\title{
Unbekanntes Kuba - Eindrücke einer botanischen Exkursion Teil 3. Der botanische Garten in Havanna
}

\author{
Hans GrasmüCK
}

\begin{abstract}
With nearly 600 hectares, the National Botanic Garden of Cuba near Havanna is the largest botanical garden in Latin America. Founded in 1968, it is characterized by its numerous woody species. Especially impressive is the palm collection. The most important plant species of Cuba are displayed. Both the garden and its herbarium contribute actively to the knowledge of the flora of Cuba.
\end{abstract}

\section{Zusammenfassung}

Der botanische Garten Havanna wurde 1968 gegründet. Mit einer Fläche von etwa 600 ha ist er der größte botanische Garten Lateinamerikas. Charakteristisch für den Garten sind seine vielen Gehölze, besonders beeindruckend ist der Palmenhain. Die wichtigsten kubanischen Arten werden hier im Garten kultiviert. Zusammen mit dem angeschlossenen Herbar werden wissenschaftliche Arbeiten zur Erforschung der endemitenreichen Flora Kubas geleistet.

\section{Die Idee des Gartens}

Auf Kuba gibt es sieben botanische Gärten, von denen der Jardín Botánico Nacional de Cuba bei Havanna der größte ist. Er ist vielleicht sogar der größte gartenarchitektonisch gestaltete Garten der Welt. Das Konzept dieses Gartens weicht etwas von demjenigen eines "typischen“ mitteleuropäischen Gartens ab. Aufgrund des beeindruckend großen Areals wird der Garten eher extensiv bewirtschaftet und gleicht einem weitläufigen Arboretum mit Baumgruppen, Palmenhainen, kleinen Wäldchen und dazwischenliegenden Wiesen. Es ist äußerst reizvoll, dass man sich an manchen Stellen des Gartens gar nicht mehr wie in einer von Menschenhand gestalteten Landschaft, sondern wie in freier Natur fühlt. Zierrabatten mit bunt blühenden Arten sind im Garten kaum angelegt, die Gewächshäuser nehmen verständlicherweise nur einen sehr geringen Teil des Gartens ein. Informationsschilder, Gartenpläne oder Namensetiketten zumindest an den markanten, größeren Bäumen sucht man leider meist vergeblich. Es ist deshalb äußerst ratsam, falls verfügbar, ein Bestimmungsbuch für kubanische Gehölze und Palmen mitzuführen (z. B. Bisse 1981, SÁNCHEZ \& LeIva 1999). Ausnahme bilden handgeschriebene Kunststoffetiketten in den AnzuchtGewächshäusern und eine eher plakative Darstellung rund um das Direktionsgebäude.

Die Erhaltung dieses Gartens in seiner bestehenden Größe und Struktur ist für Kuba ein wichtiges Anliegen. Hier in Deutschland haben wir dagegen eine andere Situation, denn die im flächenmäßigen Vergleich winzigen botanischen Gärten sind zunehmend besonders aus ökonomischen Gründen in ihrer Existenz bedroht.

Die Idee zur Gründung eines botanischen Gartens in Havanna wurde 1967 während einer Inspektionsreise der Regierung in Zentralkuba bei der Besichtigung des botanischen Gartens in Soledad bei Cienfuegos geboren. „Mach uns so etwas in Havanna, Bisse" soll der Maximo Lider Fidel Castro zu dem jungen deutschen Wissenschaftler Dr. Johannes Bisse (1935 bis 1984) gesagt haben, der 1966 mit einem Lehrauftrag als Gastdozent an der Universität Havanna nach Kuba kam (KädING 2001).

\section{Die Gartengründung}

Bereits 1968 wurde $25 \mathrm{~km}$ südlich von Havanna auf einer rund 600 ha großen Fläche, die mittlerweile vollständig kultiviert ist, der größte botanische Garten Lateinamerikas gegründet. Schon bald wurde mit den Anpflanzungen begonnen, und 1984 wurde der Garten für die Öffentlichkeit freigegeben.

1985 besuchte Fidel Castro wieder einmal den Garten und regte den Bau einiger Schauhäuser an (KädING 2001). Schon nach zwei Jahren konnten sie eingeweiht werden. Es handelt sich um vier große, teilweise miteinander verbundene Pavillons, die teils mit Glas und teils mit regendurchlässiger Schattierungsfolie 


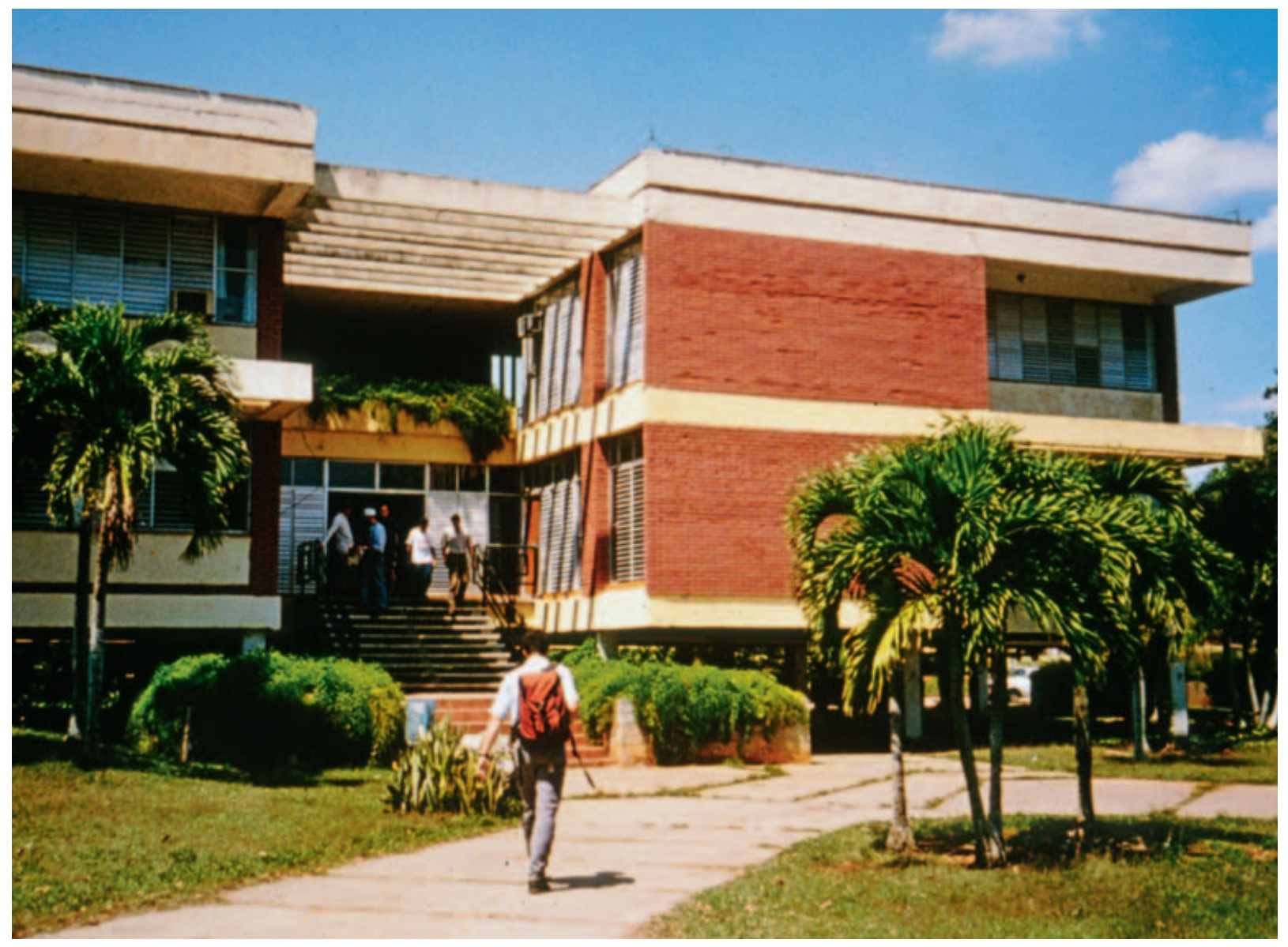

überdeckt sind. Die erste Abteilung enthält Pflanzen aus Trockengebieten Mexikos und Kubas, die nur einmal in der Woche bewässert werden. Die drei anderen Abteilungen, auch Schattenhäuser genannt, sind mit üppiger tropischer Bepflanzung versehen. Geländemodel-

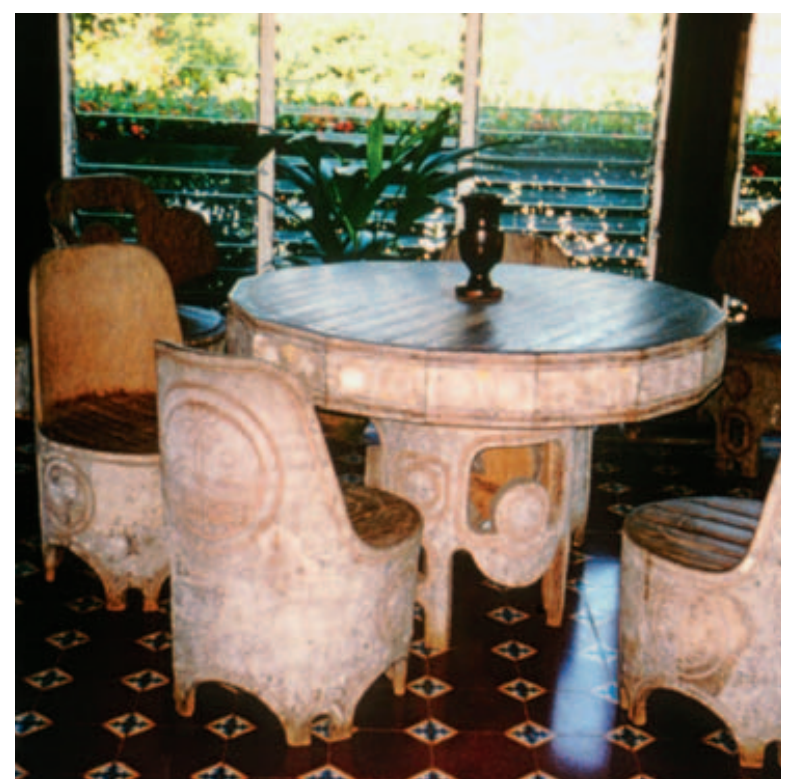

lierungen, Wasserläufe und ein kleiner Teich sorgen für natürliches Aussehen. Bei der Bepflanzung wurden vor allem Vertreter der Araceen, Begonien, Bromelien, Farne, Gesneriaceen und Orchideen verwendet. Diese Pflanzen fügen sich zu einem urwaldähnlichen Vegetationsbild zusammen.

\section{Der Pflanzenbestand}

Im Garten sind ca. 5000 Taxa vorhanden, ca. $30 \%$ davon sind in Kuba heimisch. Während eines mehrstündigen Besuches schafft der Besucher es kaum, sich alle Winkel des Gartens an-

Abb. 1 (oben): Verwaltungs- und Institutsgebäude im botanischen Garten Havanna.

Abb. 2 (unten): Möbel aus Tropenhölzern im Direktorenhäuschen.

Abb. 3 (S. 117 oben): Kleine Ausstellung über tropische Früchte.

Abb. 4 (S. 117 unten): Der Garten ist sehr weitläufig und bietet Platz für viele Palmen. 
zusehen. Denn der Garten wird von einem $35 \mathrm{~km}$ langen Netz aus asphaltierten Wegen durchzogen. Zur Besichtigung stehen deshalb drei offene, von Traktoren gezogene Besucherwagen zur Verfügung, die viermal täglich fahren. Eine Fahrt in so einem „Zug“ kann jedoch keine Führung ersetzen, da aus akustischen Gründen nur schwer zu verstehen ist, wenn der Führer Erläuterungen gibt.

Im Zentrum des Gartens liegt das 50 ha große (zum Vergleich: Größe des Palmengartens 22 ha) Palmetum mit einer Sammlung schon zu prächtigen Exemplaren herangewachsener Palmen z.B. aus den Gattungen Bactris, Coccothrinax, Colpothrinax, Copernicia, Gaussia, Roystonea, Sabalund Thrinax. Diese sind hauptsächlich in Kuba oder im karibischen Raum beheimatet. Einschließlich anderer Anlagen gibt es im Garten über 300 Palmenarten, davon mehr als 40 kubanische. Man beachte, dass allerdings der Palmengarten mit seiner im Vergleich zum kubanischen Garten winzigen Fläche eine vergleichbare Anzahl an Palmenarten kultiviert,

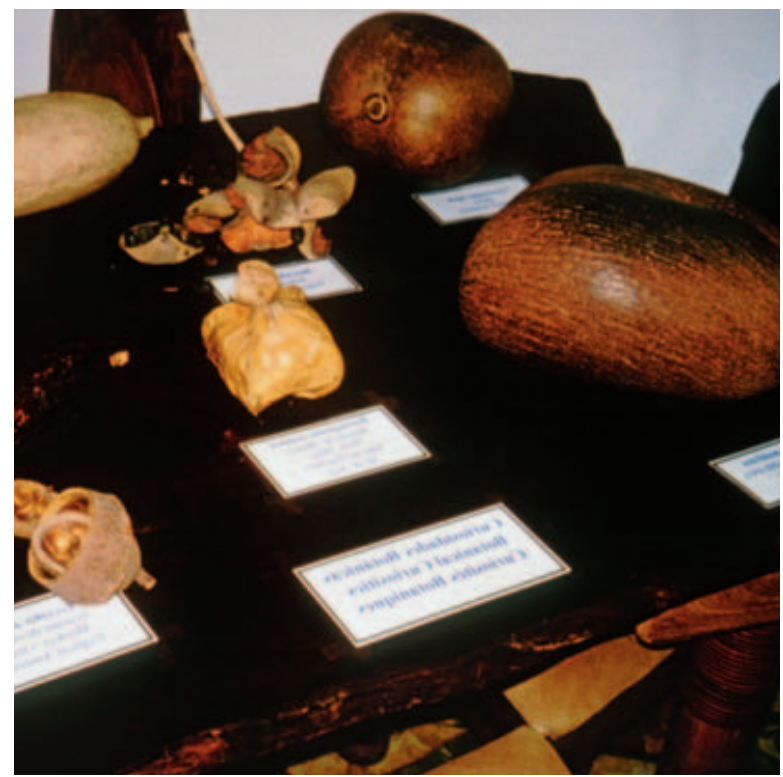

auch wenn diese natürlich oft nur kleine Pflänzchen in Töpfen und Kübeln sind.

Um das Palmetum herum sind pflanzengeographische Gruppen aus tropischen und subtropischen Gebieten angelegt. Man kann Mexiko, Zentralamerika, Südamerika (20 ha), Afrika (30 ha), Ozeanien, Kontinental- und Südost-

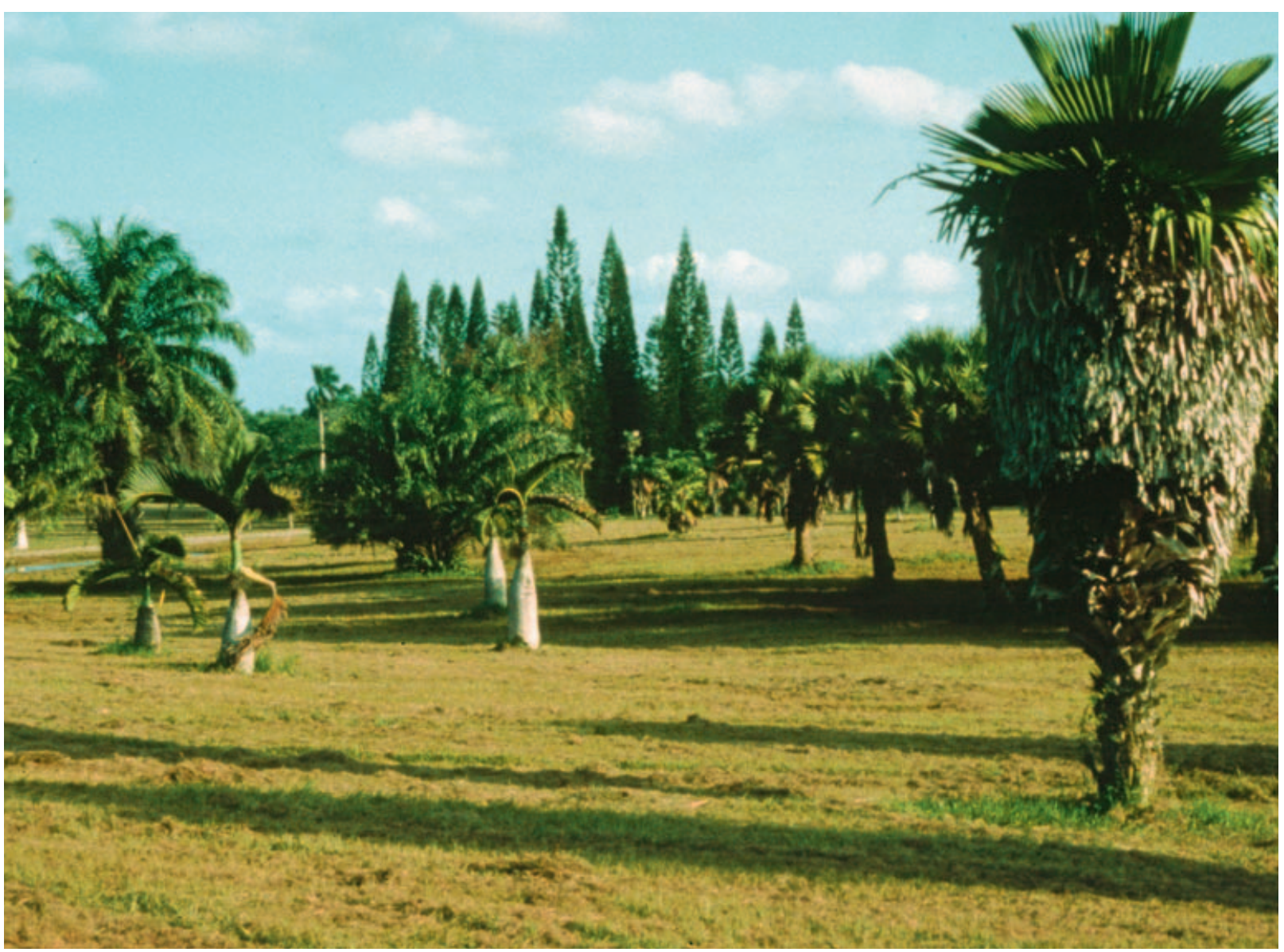




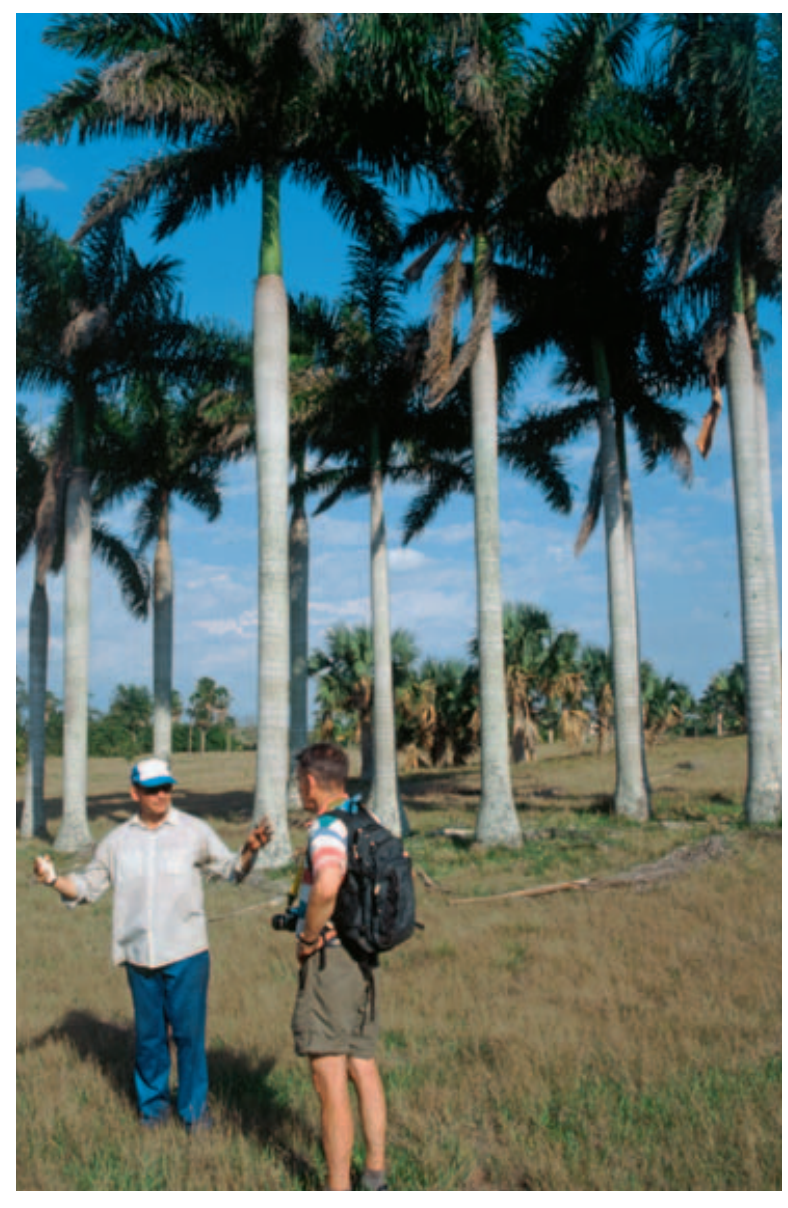

asien (25 ha) mit einem japanischen Garten (5 ha) und Australien ( 4 ha) durchqueren. Der japanische Garten wurde vom Gartenarchitekten Yoshikuni Araki geplant und konnte 1989 eröffnet werden. Zentrales Element dieses Gartens ist ein Teich, der von Lavafelsen mit Wasserfall umgeben ist. Ein Aussichtspavillon befindet sich auf einer kleinen Halbinsel, die in den Teich hereinreicht. In den japanischen Garten wurden 850 Bäume und Sträucher gepflanzt, darunter auch Podocarpus nerifolius, Ginkgo biloba, Prunus serrulata, Camelia japonica oder Nandina domestica.

Im nördlichen Teil des Gartens sind ca. 150 ha Fläche den kubanischen Pflanzen vorbehalten. Sie sind in sieben charakteristische Pflanzengesellschaften gegliedert, die in ihrer Artenzusammensetzung den natürlichen Standorten entsprechen. In jüngerer Zeit wurde ein etwa ein Hektar großes Serpentingebiet angelegt. Der ursprünglich vorhandene Mutterboden wurde ca. $1 \mathrm{~m}$ tief ausgehoben und mit einer Schicht aus 12000 Kubikmetern Serpentinboden und
Serpentinitgestein, das aus Guanabacoa nordöstlich von Havanna herangeschafft wurde, in etwa der gleichen Stärke wieder aufgefüllt. Dieses Gelände beherbergt ca. 160 endemische Arten Kubas. Sie wurden an Serpentinstandorten ausgegraben und hier angepflanzt. Alle sind sie Vertreter der Cuabales genannten, niedrigen, skleromorphen Dornbusch- und Gebüschvegetation. Diese ist an Trockenheit, Nährstoffarmut, Wasserundurchlässigkeit und Schwermetallgehalt der Böden angepasst und hat auf Kuba in verschiedenen Regionen einen bemerkenswerten Endemismus entwickelt. So gehören $75 \%$ der Arten der Cuabales der Endemitenflora Kubas an. Überhaupt spielen sklerophylle Elemente in der Flora der Antillen eine große Rolle.

Am Direktionsgebäude sind paläobotanisch interessante Pflanzen zu sehen wie verschiedene Cycadeen (z. B. Microcycas calocoma), außerdem Koniferen der Gattungen Araucaria, Cupressus, Podocarpus sowie Magnolien.

Außerdem gibt es kleinere Areale mit Kulturund Zierpflanzen, ein Experimentierfeld und eine pflanzensystematische Abteilung. Die Anzuchtgewächshäuser dienen vor allem der Vermehrung und Weiterkultur wertvoller endemischer Pflanzen Kubas, die im Garten der Erhaltungskultur zugeführt oder in die freie Natur ausgebracht werden.

\section{Mitarbeiter, Aktivitäten und Freizeitange- bote}

Weiterhin besitzt der Garten ein Sozial- und Verwaltungsgebäude sowie ein Gästehaus, in dem man sehr komfortabel übernachten kann und hervorragend verköstigt wird. Außerdem gibt es eine schön am japanischen Garten gelegene Öko-Gaststätte, zwei weitere Caféterias, in denen man vor allem frisch gepresste Fruchtsäfte zu sich nehmen kann. Von den wissenschaftlichen Einrichtungen sind eine Biblio-

Abb. 5: Der Autor dieses Beitrags während einer Führung durch den Garten.

Abb. 6 (S. 119 oben): Japanischer Garten.

Abb. 7 (S. 119 unten): Die Gewächshäuser des Gartens. 

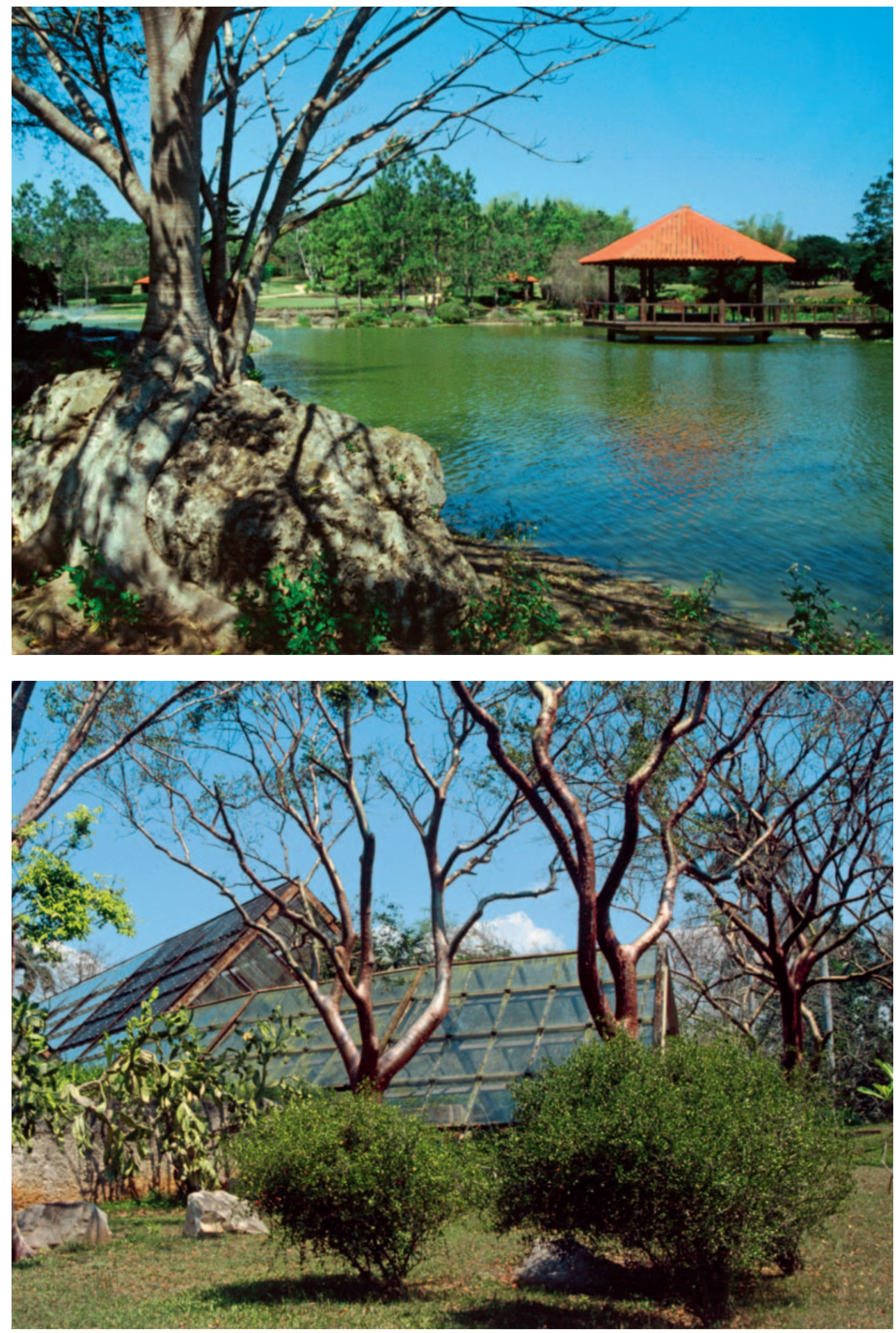

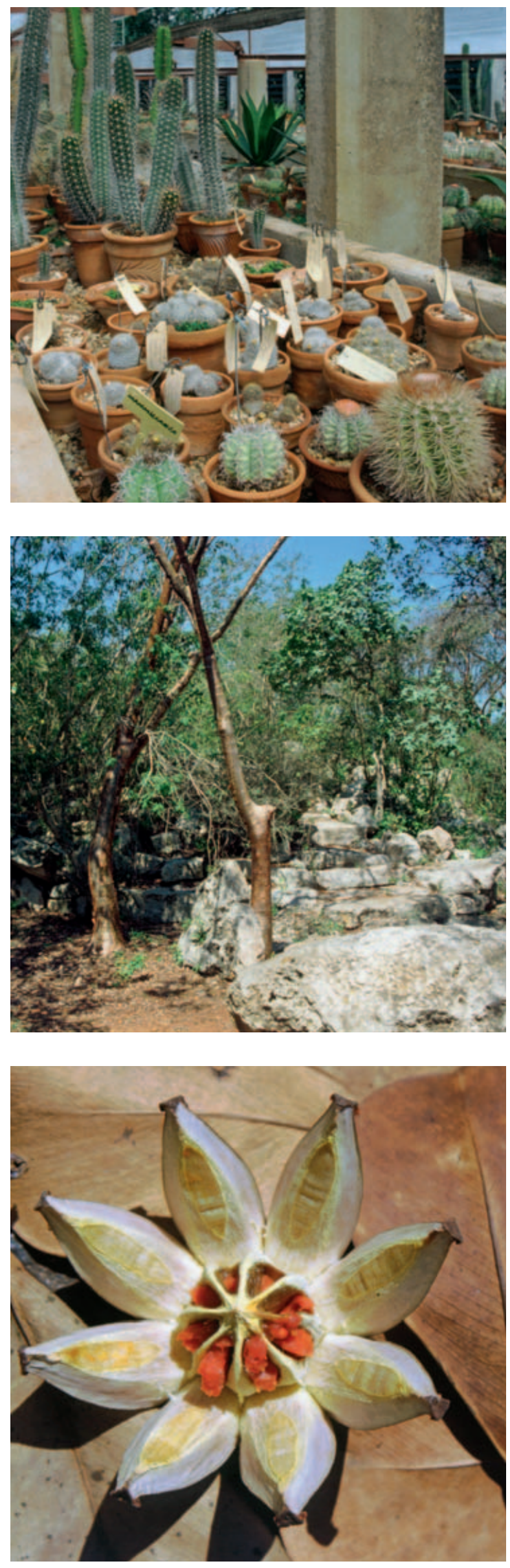

thek, Labors und Herbarien zu nennen. Im Garten sind ca. 300 Bedienstete beschäftigt, davon sind 70 ausgebildete Gärtner. 23 Personen sind wissenschaftliches Personal.

Die Mitarbeiter des Gartens bauen auf dem Gartengelände Feldfrüchte und Gemüse an, die zusammen mit den geernteten Früchten der Kulturpflanzenabteilung in der Betriebskantine verarbeitet werden. Seit Eröffnung des Gartens sind Aufzucht und Verkauf von Zierpflanzen gute Einnahmequellen. Im Jahr 1989 produzierte man rund 80000 Zierpflanzen. Weitere Geldmittel konnten durch den Export von Palmensamen erwirtschaftet werden. So wurden z. B. die Samen einer kleinwüchsigen ArecaPalme hier im Garten geerntet, geputzt und in alle Welt verschickt.

Bei der Einweihung des japanischen Gartens am 26.10.1989 sagte Fidel Castro: „Ein botanischer Garten kann nicht nur ein wissenschaftliches Zentrum sein oder eine Ansammlung von Pflanzen, ein botanischer Garten muss etwas ganz besonders Schönes sein. Von diesem Garten sollten wir die Leitgedanken, die Konzeptionen lernen, die Kunst, die Natur zusammenzustellen, wie das hier gemacht worden ist, um auf diese Weise unsere Erde immer schöner zu gestalten und unsere Stadt immer menschlicher" (KäDING 2001).

Der Garten ist täglich von 9 bis $16 \mathrm{Uhr}$ für Besucher geöffnet. Da die Entfernung zur Innenstadt von Havanna doch recht groß ist, es kein gutes Netz öffentlicher Verkehrsmittel gibt, müssen Besucher ohne Auto sich ein Taxi nehmen. Touristen können aber auch (über das Internet) einen Ausflug in den Garten buchen, wobei der Transfer vom Hotel in den Garten und zurück eingeschlossen ist. Bei der Tour wird eine 2,2 km lange Wegstrecke zurückgelegt. Die

Abb. 8 (oben): Blick in die Kakteensammlung.

Abb. 9 (Mitte): Pflanzen auf Serpentinboden.

Abb. 10 (unten): Auch auf dem Boden gibt es viel zu entdecken wie diese abgefallene Frucht von Clusia rosea.

Abb. 11 (S. 121): Ein Brotfruchtbaum in der Nutzpflanzenabteilung. 
Besucher „wandern“ dabei von Gebieten mit Küstenvegetation bis in die feuchtesten Landschaften Kubas. Die Tour endet in den Cuabales. Man kann den Tag im Garten mit einem guten Essen (Schweinebraten) ausklingen lassen. Nach Angaben aus Jahresberichten lag die jährliche Besucherzahl in den 90er Jahren bei etwa 270000 Personen, darunter auch viele Schülerinnen und Schüler.

\section{Literatur}

Bisse, J. 1981: Arboles de Cuba. - Havanna.

Käding, E. 200I: Johannes Bisse. Ein deutscher Botaniker in Kuba. - Berlin.

Jardín Botánico Nacional de Cuba (Hrsg.) 1990:

Reporte anual 1989. - Havanna.

Jardín Botánico Nacional de Cuba (Hrsg.) 1993:

Reportes anuales 1990 - 1992. - Havanna.

SÁnchez, Á. \& LeIva, T. 1999: Las palmas en Cuba. -

Havanna.

\section{Internetseiten}

http://www.casa-cuba.com7excursions-hav-

botanicalgardens.htm (abgerufen am 5.4.2006)

http://www.jgarden.org/gardesns.asp?ID=645

(abgerufen am 5.4.2006)

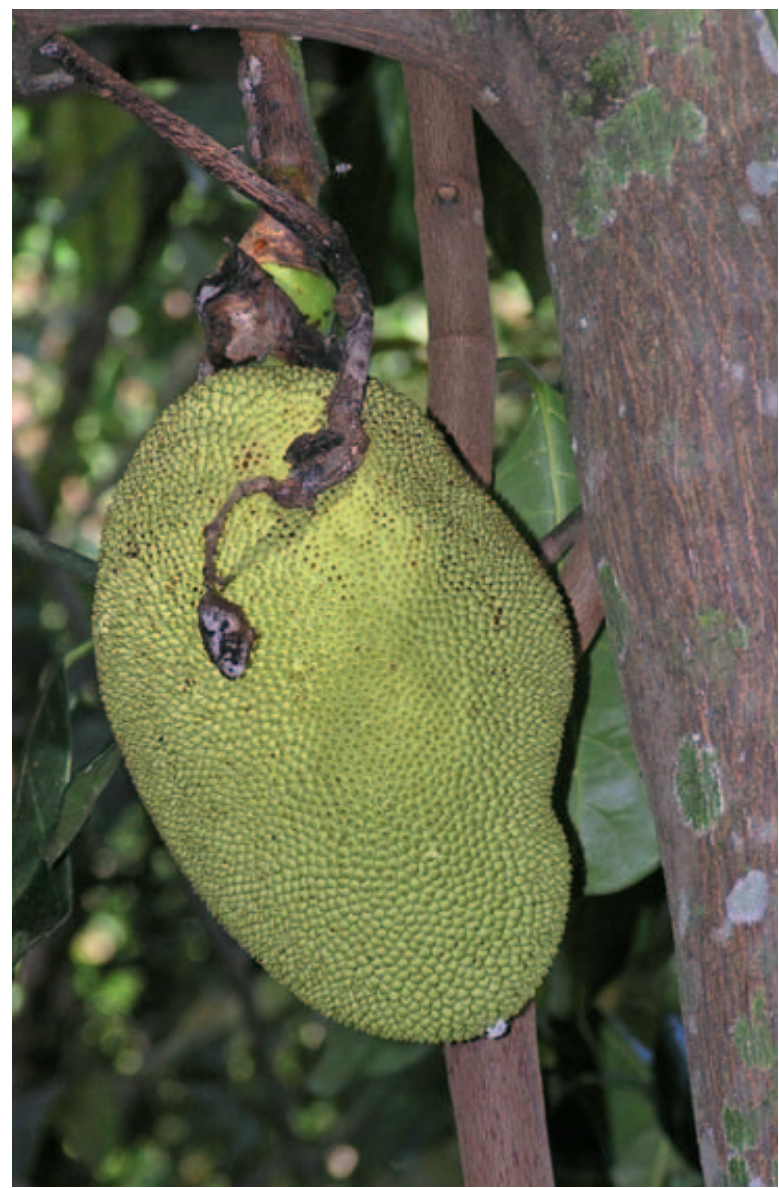

\section{Bildquellen}

Archiv Palmengarten: S. 94 (oben)

Baumann, K.: S. 106, 107, 141, 142, 143, 144 (oben)

BOGNER, J.: S. 129

Gerlach, G.: S. 130, 131 (oben)

Firsov, G.: S. 110, 111, 112, 113

Freye, R.: S. 152, 167

GoERKe (1989): S. 97

http://bibbild.abo.fi/Linneana/linkat.htm: S. 96 (oben) http://bibbild.abo.fi/Linneana/pk1.htm: S. 96 (unten)

NADOLNY, W.: S. 147
JaCobi, M.: S. 161 (unten), 164

Schwarzmeier, N.: S. 116, 117, 120 (oben, Mitte)

Steinecke, H.: S. 88, 89, 90, 91, 92, 93, 94 (unten), 99, 100, 101, 102, 103 (oben), 118, 120 (unten), 121, 135, $136,137,138,139,145,146,149,154,155,156,158$, 159, 160, 161 (oben), 162, 163

Steinecke, K.: S. 103 (Mitte, unten), 104, 118, 120 (unten), 121, 144 (unten), 165

ThyMakis, N.: S. 150, 151

upload.wikimedia.org: S. 98

ZAHORKA, H.: S. 123, 124, 125, 126, 127, 128, 133 (oben, Mitte) 